\title{
Design of the Wendelstein 7-X Inertially Cooled Test Divertor Unit Scraper Element
}

\author{
Arnold Lumsdainea, Jean Boscaryb ${ }^{b}$, Joris Fellingerc, Jeff Harris ${ }^{a}$, Hauke Hölbec, Ralf Königc, Dean \\ McGinnisa, Jeremy Lore ${ }^{a}$, Hutch Neilsond, Peter Titus ${ }^{d}$, Jörg Tretterb \\ ${ }^{a}$ Oak Ridge National Laboratory, Oak Ridge, Tennessee, USA \\ ${ }^{b}$ Max Planck Institute for Plasma Physics, Garching, Germany \\ ${ }^{c}$ Max Planck Institute for Plasma Physics, Greifswald, Germany \\ ${ }^{d}$ Princeton Plasma Physics Lab, Princeton, New Jersey, USA
}

\begin{abstract}
The Wendelstein 7-X stellarator is scheduled to begin operation in 2015, and to achieve full power steady-state operation in 2019. Computational simulations have indicated that for certain plasma configurations in the steadystate operation, the ends of the divertor targets may receive heat fluxes beyond their qualified technological limit. To address this issue, a high heat-flux "scraper element" (HHF-SE) has been designed that can protect the sensitive divertor target region. The surface profile of the HHF-SE has been carefully designed to meet challenging engineering requirements and severe spatial limitations through an iterative process involving physics simulations, engineering analysis, and computer aided design rendering. The desire to examine how the scraper element interacts with the plasma, both in terms of how it protects the divertor, and how it affects the neutral pumping efficiency, has led to the consideration of installing an inertially cooled version during the short pulse operation phase. This Test Divertor Unit scraper element (TDU-SE) would replicate the surface profile of the HHF-SE. The design and instrumentation of this component must be completed carefully in order to satisfy the requirements of the machine operation, as well as to support the possible installation of the HHF-SE for steady-state operation.
\end{abstract}

\section{Wendelstein 7-X, stellarator, plasma facing component, divertor, high heat flux}

\section{Introduction}

The divertor of the W7-X stellarator will evolve through three distinct phases of operation. For the first plasma, scheduled to begin in 2015, the device will operate with a limiter instead of a divertor. For the next operational phase, scheduled to begin in 2016, an inertially cooled divertor (called the Test Divertor Unit, or TDU) will be installed so that the device can operate at higher power for short pulses. Finally, an actively cooled divertor will be in place for steady-state, full power operation, scheduled for 2019. This high heatflux divertor will use a flat tile technology that is qualified to handle heat fluxes of $10 \mathrm{MW} / \mathrm{m}^{2}$ [1]. However, the edges of these tiles are qualified to a lower limit of $5 \mathrm{MW} / \mathrm{m}^{2}$.

Plasma simulations have shown that a bootstrap current of up to 43kA may evolve for certain plasma configurations. This could cause the edges of the divertor targets to be overloaded. A high heat-flux scraper element (HHF-SE), if placed in front of the divertor pumping gap, would intercept a portion of the convective flux and protect the divertor edges (see Fig. 1) [2]. This HHF-SE has been designed using carbon This manuscript has been authored by UT-Battelle, LLC, under Contract No. DE-AC05-00OR22725 with the U.S. Department of Energy. The United States Government retains and the publisher, by accepting the article for publication, acknowledges that the United States Government retains a non-exclusive, paid-up, irrevocable, world-wide license to publish or reproduce the published form of this manuscript, or allow others to do so, for United States Government purposes.

This project has received funding from the European Union's Horizon 2020 research and innovation programme under grant agreement number 633053. The views and opinions expressed herein do not necessarilv reflect those of the Eurodean Commission. fiber reinforced carbon composite monoblocks which have been shown to withstand steady-state heat fluxes up to $20 \mathrm{MW} / \mathrm{m}^{2}$ [3-4]. A HHF-SE would be placed in each of the ten W7-X divertor locations.

There are two primary questions regarding the inclusion of the HHF-SE during steady-state operation. First, will it protect the divertor edges as predicted in simulation? Second, will the HHF-SE detrimentally affect the neutral pumping efficiency? In order to answer these questions, the inclusion of an inertially cooled Test Divertor Unit scraper element (TDU-SE) has been planned for the short pulse operational phase.

\section{Physics simulation}

The bootstrap current that produces the unacceptable heat fluxes for which the HHF-SE was developed evolve over the time scales of roughly 30 seconds. The time scales for short pulse operation (TDU phase) will be on the order of 10 seconds, thus it will not be possible to create a plasma scenario with this bootstrap current that will appropriately load the TDU-SE. A set of magnetic coil currents that produce a vacuum field configuration that mimic the scenarios of concern have been developed. A 22kA-mimic and a 43kA-mimic configuration have been planned. The convective heat fluxes on the divertor components have been modeled using magnetic field configurations [5-6]. The strike patterns and heat fluxes that occur on the divertor target and the scraper element are shown in Fig. 2. The heat flux that results from the 22kA bootstrap current during long pulse operation is compared with the $22 \mathrm{kA}$-mimic 
configuration during short-pulse operation. It is believed that the resulting flux stripes are qualitatively similar enough to draw conclusions during short pulse operation about the use of the scraper element in long pulse operation. However, the TDU-SEs will be placed in only two of the ten divertor locations. Simulations have shown that this will result in up to $40 \%$ higher load on these components. It is expected that reducing the input power into the device can compensate for this without compromising the measurements needed to assess the effects of the presence of the scraper element.

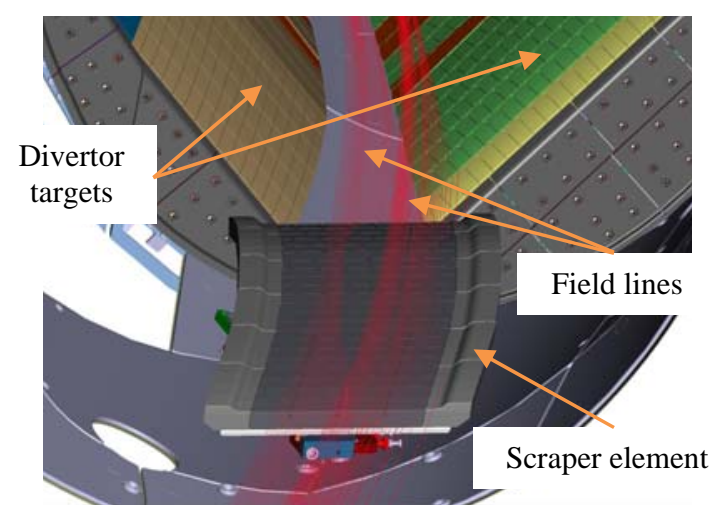

Fig. 1. View of W7-X divertor region with field lines.

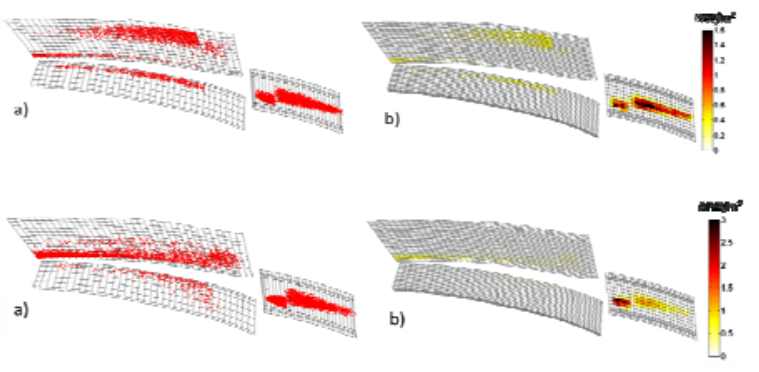

Fig. 2. Strike patterns (a) and heat flux (b) for $22 \mathrm{kA}$ bootstrap current configuration (top) and 22kA-mimic configuration (bottom).

\section{Design}

The design of the scraper element has been done from the "top-down" with the plasma facing surface being the most critical element. The HHF-SE is the design basis for the TDU-SE, where having an equivalent top surface is a requirement for the design. Fig. 3 shows the similarities and differences between the HHF-SE and TDU-SE full assemblies along with the support base mount.

\subsection{Requirements}

Some of the requirements on the design of the TDUSE can be described as follows:

- Maximum surface temperature of graphite $=1800^{\circ} \mathrm{C}$.

- Maximum surface temperature of wall panels behind the TDU-SE $=200^{\circ} \mathrm{C}$.

- Maximum temperature of stainless steel support components $=500^{\circ} \mathrm{C}$.
- Magnetic permeability < 1.01 for all materials.

- Cobalt content $<500$ ppm for all parts.

- Assembly can be adjusted $+/-7 \mathrm{~mm}$ in each direction during installation.

- The final position of the assembly must be within +/$1.5 \mathrm{~mm}$ in any direction of the target position.

- TDU-SE base plate structure can accept $100 \mathrm{~kg}$ load .

- TDU-SE can be installed from pieces weighing less than $25 \mathrm{~kg}$.

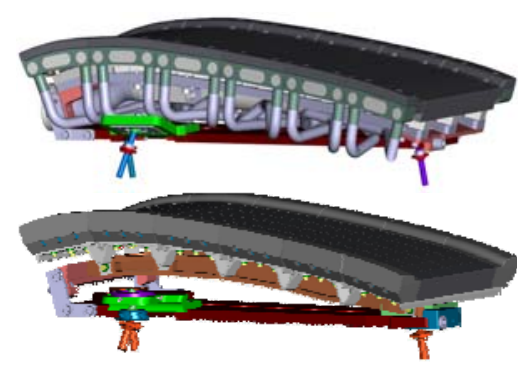

Fig. 3. Conceptual designs of HHF-SE (top) and TDUSE (bottom)

Operation requirements will be satisfied under a design basis scenario, which is similar to the design bases for the TDU. A 1.25 MJ heat load will be applied, which is expected to be a rectangular heat pattern with a peak of $8 \mathrm{MW} / \mathrm{m}^{2}$ (decreasing linearly over a $5 \mathrm{~cm} \mathrm{x}$ $67.5 \mathrm{~cm}$ region) for 10 seconds.

In order to accomplish the mission of the TDU-SE, the following instruments will be necessary. Thermocouples will measure the temperature in the graphite tile and on the support structure. These will be used to confirm that temperatures are below operational limits, and in conjunction with finite element simulation, estimate the heat load on the TDU-SE surface. Infrared (IR) cameras will measure the temperature profile on the surface of the TDU-SE, which will give an idea of the shape and magnitude of the heat load. Neutral pressure gauges in the pumping gaps, as well as beneath a TDUSE, will gauge how pumping effectiveness is affected by the presence of the scraper elements. It is also planned to include Langmuir probes, and an $\mathrm{H}_{\alpha}$ camera.

\subsection{Mounting locations}

The scraper element was not planned for installation when the in-vessel components were originally designed, and many of the components have already been installed. Thus, it has been planned to mount the TDU-SE on existing supports that are currently used to mount the stainless steel wall panels which protect the plasma vessel from radiative heat [7]. Fig. 4 shows several wall panels in front of the divertor region, including three panel supports that are planned for mounting the TDUSE. The panels beneath the TDU-SE will still be kept in place, and a mounting screw will be placed into the panel supports. This provides several challenges, both in terms of available space, as well as stability for the installation and operation of the component. Two different types of supports are used for the wall panels - 
one design is fixed in place, and the other allows some movement, in order for the panel's thermal expansion to not be overly restrained when it is heated. The TDU-SE requires that two of the three mount locations be a fixed support, while the other one can be the flexible design. Fig. 5 shows a top view of the base plate for the TDUSE. Given that the final mounting position must be within $+/-1.5 \mathrm{~mm}$ of the target position, the mounting locations must have sufficient adjustment capability. But they also must not allow the plasma facing surface to move once the installation is complete. Finally, they must allow for thermal expansion in the base plate during operation. The degrees-of-freedom that are free in each mount location are shown in Fig. 5. Fig. 6 shows the design of the connections to the panel mount, along with the design of one of the panel mounts that it will screw into. In addition, rotations abound the ball joint at the top of the screw are free to allow for bowing of the TDU-SE without stressing this joint.

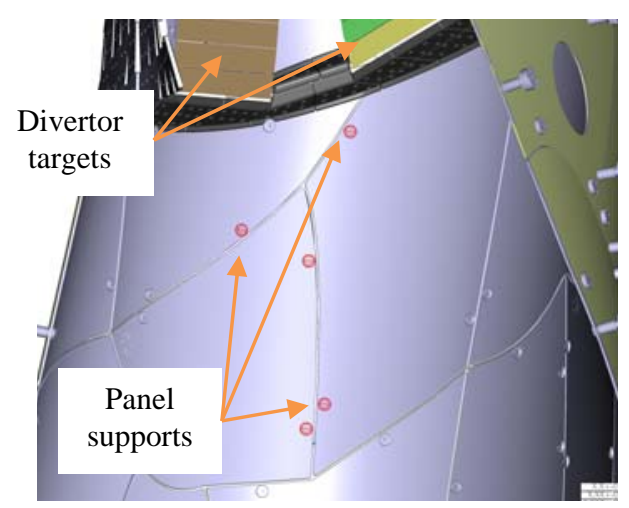

Fig. 4. Conceptual designs of HHF-SE (top) and TDUSE (bottom)

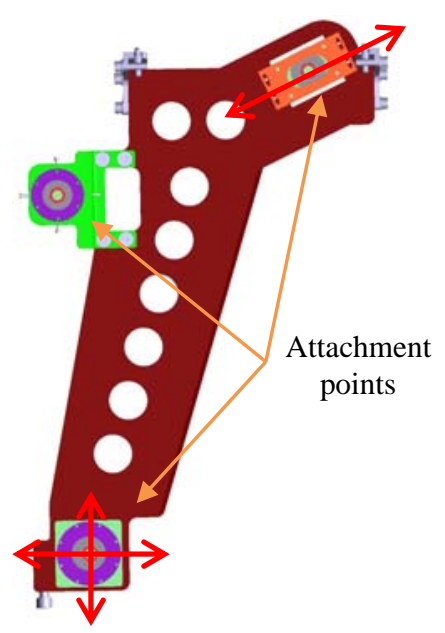

Fig. 5. Base plate of TDU-SE with attachment points and degrees-of-freedom

\subsection{Plasma facing surface}

The top surface of the TDU-SE will be created by assembling six graphite modules, each module (see Fig. 7) geometrically mimicking the actively cooled modules of the HHF-SE. Each module will be manufactured to high tolerance to prevent leading edges. Each module also includes $0.5 \mathrm{~mm}$ wide cuts (approximately $10 \mathrm{~mm}$ deep and $60 \mathrm{~mm}$ apart) to relieve thermal stresses. These will then be mounted onto a support truss to complete the plasma facing assembly (see Fig. 8). This will be assembled and carefully measured outside of the vessel, and then installed as one piece onto the base plate.

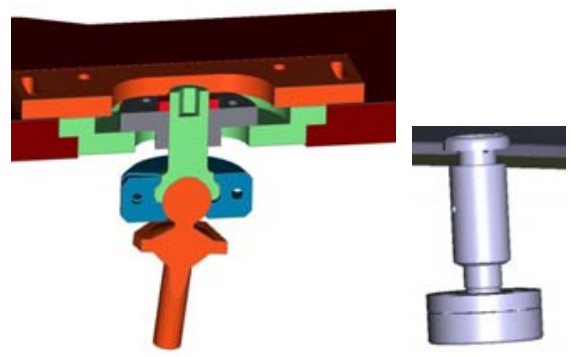

Fig. 6. Cross-section of connection device, and image of panel support

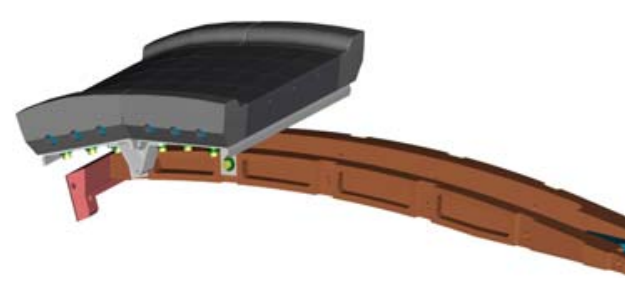

Fig. 7. TDU-SE single graphite module mounted on stainless steel support truss

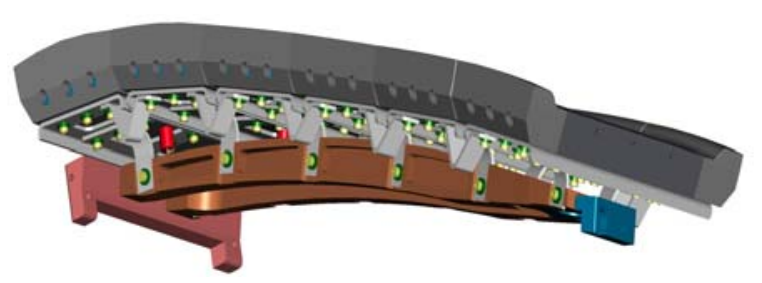

Fig. 8. TDU-SE truss and module assembly

\section{Analysis}

Preliminary finite element analyses have been performed in order to consider whether the various components will remain under the thermal requirements outlined previously, and to examine the stress in the graphite tiles. Fig. 9 shows the thermal result of a single tile, loaded with an $8 \mathrm{MW} / \mathrm{m}^{2}$ heat flux over a $100 \mathrm{~mm}$ wide stripe for 6.5 seconds. It can be seen that the top surface is above the $1800{ }^{\circ} \mathrm{C}(2073 \mathrm{~K})$ limit. But this heat load has twice the specified width (and thus more than twice the integrated heat, as the specified heat load is not constant over the area, tails off linearly from the peak). Stress results also indicate that the graphite may be near its tensile strength limit in the circular regions at the bottom of the relief cuts. Further analyses, which more accurate application of heat flux, are under way. As the plasma scenarios that will load the TDU-SE are 
not the standard operational scenarios, it will be possible to reduce the input power or to increase the rest time between shots in order to keep the temperatures and stresses within material limits. It will be necessary when operation begins to diagnose how closely the actual plasma interaction with the TDU-SE matches the simulation.

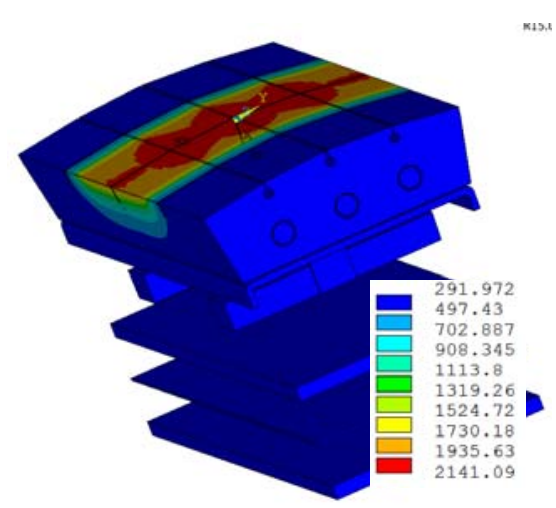

Fig. 9. Temperature (in $\mathrm{K}$ ) result from $8 \mathrm{MW} / \mathrm{m}^{2}$ heat flux on graphite module.

Fig. 10 shows the results of a $1 \mathrm{D}$ analysis of the various components in the TDU-SE. An $8 \mathrm{MW} / \mathrm{m}^{2}$ heat flux is applied over a $100 \mathrm{~mm}$ wide stripe for 6.25 seconds, with a 10 minute rest time between each shot. Radiation to the vessel with an emissivity of 0.4 is included in the model. Three thermal shields are also included beneath the base plate in order to prevent the wall panel from exceeding its temperature limit. Several features can be noted from this analysis. First, there is significant ratcheting predicted in this configuration. This is not surprising given that radiation is the only method of heat dissipation. Second, both the graphite and the wall panel exceed their thermal limit $\left(1800{ }^{\circ} \mathrm{C}\right.$ and $200{ }^{\circ} \mathrm{C}$, respectively). However, although further analysis is required, these results are not a concern. It is believed that this is an overly conservative analysis, as the emissivity is likely to be closer to black body emission. Also, there will be radiation losses from the sides of the component which are not included in the 1D analysis. A full 3D analysis is quite complex, but is in process [8]. Furthermore, the rest time between shots could be lengthened, or different plasma scenarios that do not engage the scraper element could be run in between plasma shots to test the TDU-SE in order to allow the components to cool.

\section{Future plans}

Two TDU-SE complete assemblies are planned for manufacture and delivery by mid-2016. They will be installed during a planned shut-down in the middle of the short pulse operation at the start of 2017. It will then be possible to compare the pumping efficiency before and after the TDU-SEs are installed. Comparisons also will be made between the divertor areas that have a TDU-SE installed with those that do not. It is planned to install one TDU-SE above the equator of the torus, and one below. It will also be possible to examine the up-down asymmetry of the plasma operation.

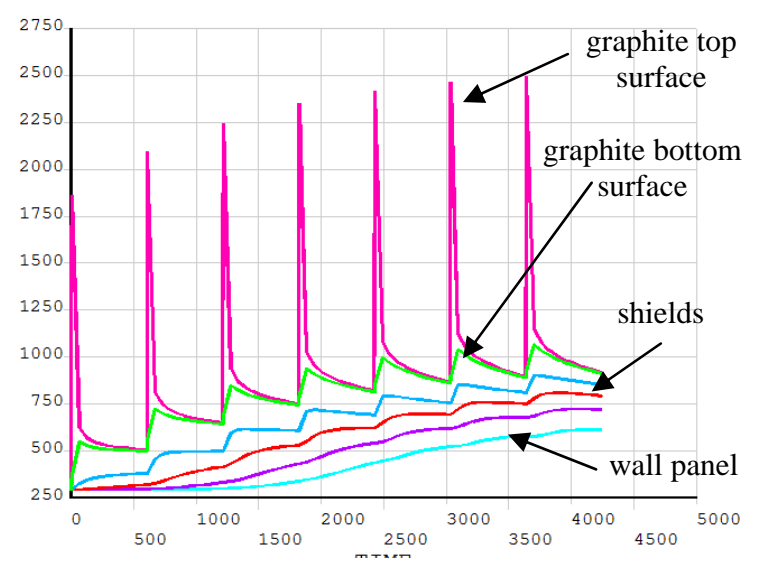

Fig. 10. Temperature (K) vs. time from finite element thermal result.

It is also planned to deliver a mock-up TDU-SE in mid-2016. The purpose of building a mock-up of the TDU-SE is to give opportunity to gain experience in the process of assembling and installing the TDU-SE in a sample vacuum vessel sector prior to the actual installation. Plans are to deliver a prototypic base plate and support truss. The mock-up top surface will be made of an inexpensive, easily machinable material, and will not require the surface to be manufactured to the same precision as the graphite block.

\section{References}

[1] A. Peacock, et al, Wendelstein 7-X high heat flux components, Proceedings of the 2013 Symposium on Fusion Engineering, San Francisco, CA, 2013.

[2] J. Boscary, et al, Development activities of the high heat flux scraper element, Fus. Eng and Des., these proceedings.

[3] M. Richou, et al, Assessment of CFC grades under thermal fatigue for the ITER inner vertical target, Physica Scripta, T145, (2011) 1-5.

[4] A. Lumsdaine, et al, Design and analysis of the W7-X divertor scraper element, Fus. Eng and Des., 88, (2013) 1773-1777.

[5] J. Lore, et al, Design and analysis of divertor scraper elements for the W7-X stellarator, IEEE Transactions on Plasma Science, 42, (2014) 539-544.

[6] J. Lore, et al, Heat flux and design calculations for the W7-X divertor scraper element, Proceedings of the 24th IAEA Fusion Energy Conference, San Diego, CA, (2012).

[7] A. Peacock, et al, The procurement and testing of the stainless steel in-vessel panels of the Wendelstein 7-X Stellarator, Fus. Eng and Des. 86, (2011) 1706-1709.

[8] P. Titus, Analysis of the Wendelstein 7-X Inertially Cooled Test Divertor Unit Scraper Element, Fus. Eng and Des., these proceedings. 